\title{
Prognostic significance of MYCN related genes in pediatric neuroblastoma: a study based on TARGET and GEO datasets
}

\author{
Haiwei Wang ${ }^{1 * \dagger} \mathbb{D}$, Xinrui Wang ${ }^{1 \dagger}$, Liangpu Xu' ${ }^{1}$, Zhang $^{2^{*}}$ and Hua Cao ${ }^{1 *}$
}

\begin{abstract}
Background: Neuroblastoma patients with MYCN amplification are associated with poor prognosis. However, the prognostic relevance of MYCN associated genes in neuroblastoma is unclear.

Methods: The expression profiles of MYCN associated genes were identified from Therapeutically Applicable Research to Generate Effective Treatments (TARGET) and Gene Expression Omnibus (GEO) datasets. Enriched transcription factors and signaling pathways were determined using gene set enrichment analysis (GSEA). KaplanMeier plotter was used to identify the prognostic relevance of MYCN associated genes. Multivariate cox regression and Spearman's correlation were used to determine the correlation coefficients of MYCN associated genes.

Results: In TARGET and GSE85047 datasets, neuroblastoma patients with MYCN amplification were associated with worse prognosis. Transcription factor MYC was positively associated with MYCN amplification in GSEA assay. We identified 13 MYC target genes which were increased in neuroblastoma patients with MYCN amplification in TARGET, GSE19274 and GSE85047 datasets. Moreover, six out of the 13 MYC target genes ARMC6, DCTPP1, EIF4G1, ELOVL6, FBL and PRMT1 were associated with adverse prognosis in TARGET and GSE85047 datasets. Transcription factor E2F1 was up-regulated by MYCN amplification and associated with the poor prognosis of neuroblastoma. Furthermore, RPS19 in ribosome signaling pathway was also associated with MYCN amplification and correlated with the poor prognosis of neuroblastoma. At last, we showed that most of MYCN target genes were correlated with each other. However, EIF4G1 was an independent prognostic marker. And the prognostic effects of the combination of MYCN amplification and EIF4G1 expression were more significant than MYCN or EIF4G1 alone.

Conclusions: MYCN target genes ARMC6, DCTPP1, EIF4G1, ELOVL6, FBL, PRMT1, E2F1 and RPS19 had significant prognostic effects in pediatric neuroblastoma. And neuroblastoma patients without MYCN amplification and low EIF4G1 expression had best prognosis.
\end{abstract}

Keywords: Pediatric neuroblastoma, MYCN, E2F1, EIF4G1, Ribosome signaling pathway, TARGET, GEO

\footnotetext{
* Correspondence: hwwang@sibs.ac.cn; Zj11222@rjh.com.cn;

caohua69@fjmu.edu.cn

†Haiwei Wang and Xinrui Wang contributed equally to this work.

${ }^{1}$ Medical Research Center, Fujian Maternity and Child Health Hospital,

Affiliated Hospital of Fujian Medical University, Fuzhou, Fujian, China

${ }^{2}$ State Key Laboratory for Medical Genomics, Shanghai Institute of

Hematology, Rui-Jin Hospital Affiliated to School of Medicine, Shanghai Jiao

Tong University, Shanghai, China
}

(c) The Author(s). 2020 Open Access This article is licensed under a Creative Commons Attribution 4.0 International License, which permits use, sharing, adaptation, distribution and reproduction in any medium or format, as long as you give appropriate credit to the original author(s) and the source, provide a link to the Creative Commons licence, and indicate if changes were made. The images or other third party material in this article are included in the article's Creative Commons licence, unless indicated otherwise in a credit line to the material. If material is not included in the article's Creative Commons licence and your intended use is not permitted by statutory regulation or exceeds the permitted use, you will need to obtain permission directly from the copyright holder. To view a copy of this licence, visit http://creativecommons.org/licenses/by/4.0/ The Creative Commons Public Domain Dedication waiver (http://creativecommons.org/publicdomain/zero/1.0/) applies to the data made available in this article, unless otherwise stated in a credit line to the data. 


\section{Background}

Neuroblastoma is a common pediatric solid tumor derived from the sympathetic nervous system and a major cause of pediatric cancer related mortality $[1,2]$. Neuroblastoma is highly heterogeneous and the prognosis of neuroblastoma is highly variable. Some neuroblastoma rapidly regresses with standard treatment, while, other neuroblastoma progresses despite the extensive treatment [3]. Age, tumor stage [4], tumor ploidy [5], Aurora kinase A expression [6], hypoxia gene signature [7] and RAS and/or TP53 mutations [8] are all used for the prediction of the clinical outcomes of neuroblastoma. However, new prognostic markers are still needed.

MYCN amplification represents the strongest independent adverse prognostic factor [9]. MYCN belongs to the MYC transcription factor family and is amplified in approximate $25 \%$ of neuroblastoma patients [10]. According to the International Neuroblastoma Risk Group, neuroblastoma patients are classified into low, intermediate or high risk subgroups based on MYCN amplification status [11]. Patients with MYCN amplification are associated with high risk and worse prognostic outcome [12]. Except MYCN amplification, MYCN protein expression [13], MYCN target gene CD44 [14] and MYCN signature [15] are used to predict the outcome of neuroblastoma. As a master transcription factor, MYCN controls the transcriptional activity of multiple target genes [16]. However, the globe MYCN amplification regulated genes and their predictive relevance in neuroblastoma are unclear.

In the present study, using published TARGET [17] and GEO datasets, we comprehensively analyzed the differentially expressed genes in neuroblastoma patients with or without MYCN amplification, and identified the critical signaling pathways and transcription factors involving MYCN regulation. We also analyzed the prognostic effects of MYCN target genes in pediatric neuroblastoma. Overall, we found eight MYCN target genes ARMC6, DCTPP1, EIF4G1, ELOVL6, FBL,
PRMT1, E2F1 and RPS19 which had significant prognostic effects in pediatric neuroblastoma patients.

\section{Methods \\ Data collection}

The clinical and expression datasets of neuroblastoma patients were downloaded from the TARGET project, launched by national cancer institute (https://ocg.cancer. gov/). Expression series matrix of neuroblastoma tissues was also downloaded from GEO website (https://www. ncbi.nlm.nih.gov/geo/), including GSE19274, GSE73517, GSE49710 and GSE85047 datasets.

\section{Prognostic effects of MYCN amplification}

Clinical data of neuroblastoma patients deposited in TARGET and GSE85047 was used to determine the different overall survival of neuroblastoma patients with or without MYCN amplification. Log-rank test was used to determine the $P$ values.

\section{GEO data processing}

The gene expression matrix was annotated with corresponding platform. The expression values were processed using $R$ software ( $\mathrm{R}$ version 3.5.0) "plyr" package (version 1.8.5; https://cran.r-project.org/web/packages/plyr/index.html).

\section{Gene set enrichment analysis (GSEA)}

Signaling pathway and transcription factor enrichment was performed using GSEA 2.0 software (http://www. broad.mit.edu/gsea/index.html). Statistical significance $P$ values were determined by 1000 gene set permutations.

\section{Heatmap presentation}

Heatmaps were generated by "pheatmap" package (version 1.0.12; https://cran.r-project.org/web/packages/ pheatmap/ index.html) in $\mathrm{R}$ software. The clustering scale was determined by "average" method. The clustering distance was determined by the 'correlation' method.
TARGET

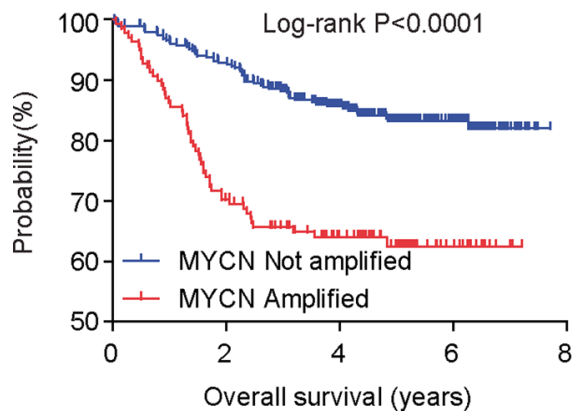

GSE85047

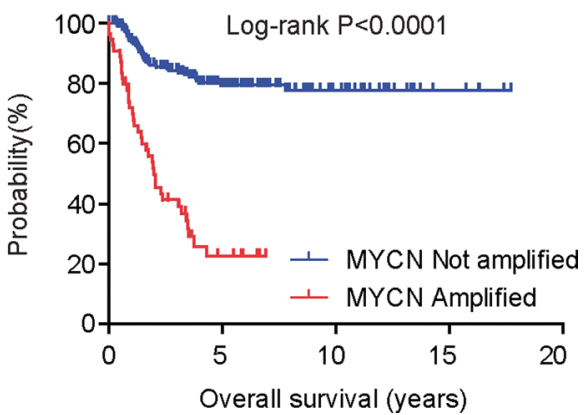

Fig. 1 Prognostic significance of MYCN amplification in patients with neuroblastoma. Kaplan-Meier plots showed the prognostic relevance of MYCN amplification in patients with neuroblastoma in both TARGET and GSE85047 datasets. Different overall survival between patients with (red) or without (blue) MYCN amplification was determined by log-rank test 
a

Enrichment plots of MYC transcription factor
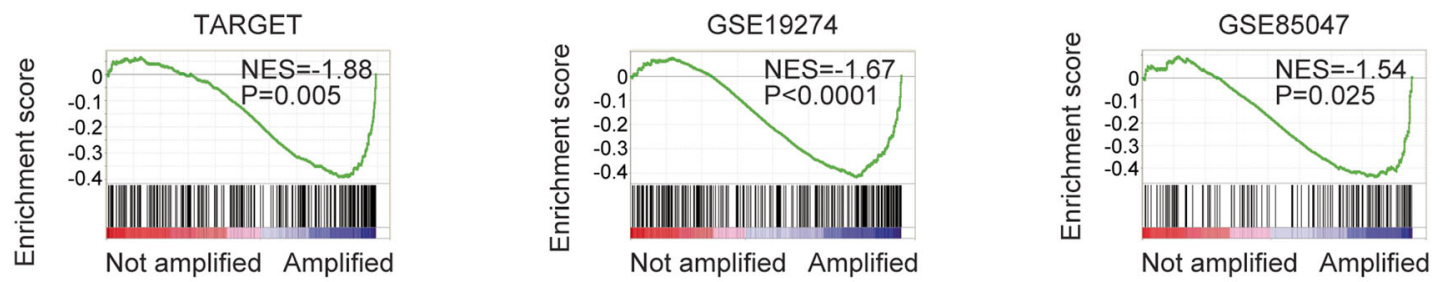

b

TARGET
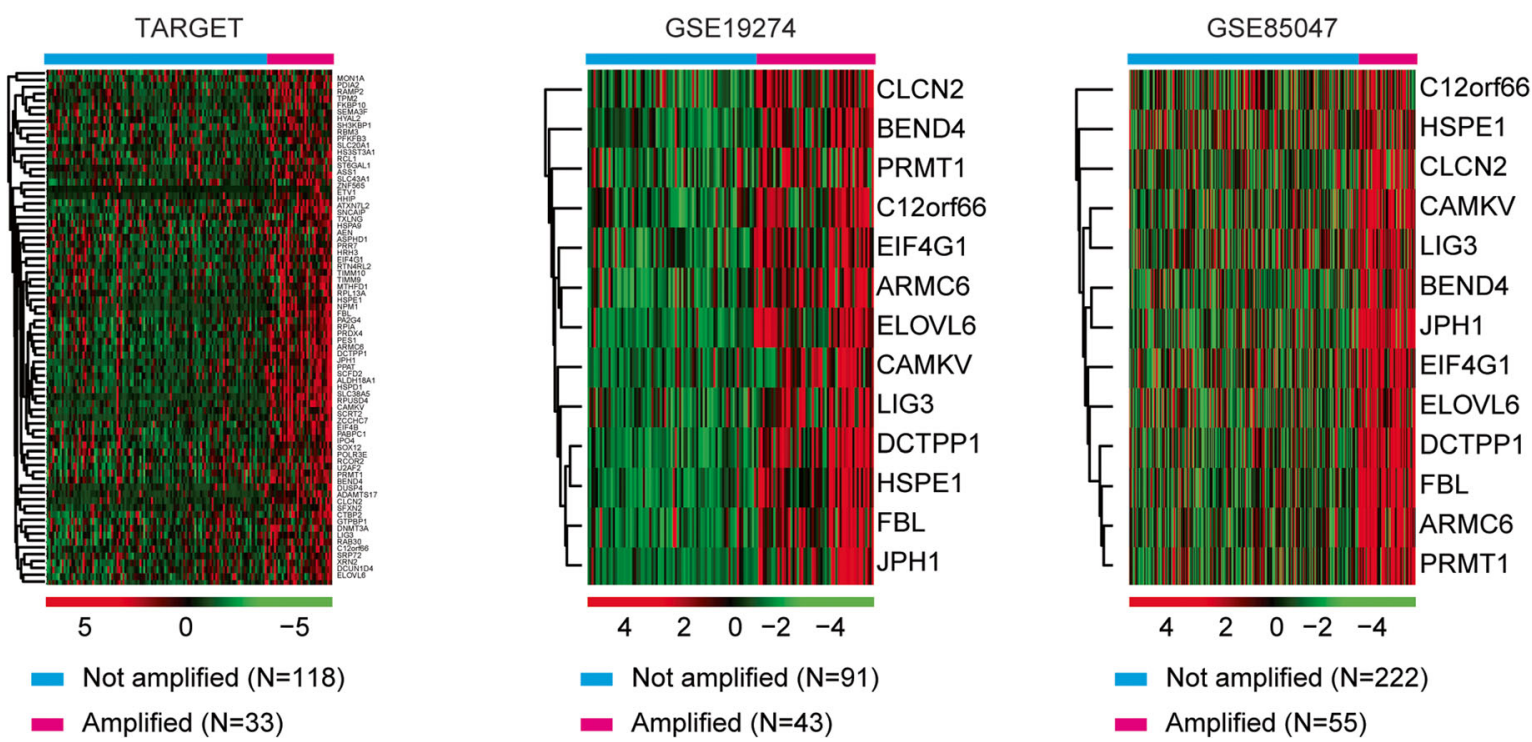

Fig. 2 Identification of MYCN target genes in patients with neuroblastoma. a Enrichment plots of transcription factor MYC in TARGET, GSE19274 and GSE85047 datasets. Enrichment of normalized enrichment score (NES) and P-values were presented. $\mathbf{b}$ Heatmaps demonstrated the expression profiles of MYC target genes in MYCN amplified and not amplified neuroblastoma tissues in TARGET, GSE19274 and GSE85047 datasets. Genes up-regulated (red), down-regulated (blue) and moderately regulated (black) were delineated

\section{Prognostic effects of MYCN target genes}

'Survival' package (version 3.1-8; https://cran.r-project. org/web/packages/survival/ index.html) in $\mathrm{R}$ software was used to determine the clinical influence of MYCN target genes. The neuroblastoma patients were divided into two subgroups based on the mean expression levels of MYCN target genes. Log-rank test was used to test the different clinical outcomes in neuroblastoma patients with high expression levels and low expression levels of MYCN target genes.

\section{Multivariate cox regression}

$\mathrm{R}$ software 'survival' package (version 3.1-8) was used for multivariate cox regression analysis. Log-rank test was used to calculate the $P$ values based on 'coxph' method.

\section{Correlation plots of MYCN target genes}

Correlation plots of MYCN target genes were created using the 'corrplot' package (version 0.84; https://cran.r- project.org/web/packages/corrplot/index.html) in R software. The correlation coefficients were determined by Spearman's correlation test.

\section{Statistical analysis}

The box plots were generated from GraphPad Prism software (version 5.0; https://www.graphpad.com/). Statistical analysis was performed using the Student's t test using $\mathrm{R}$ software. $P$ value less than 0.05 was chosen to be statistically significant difference.

\section{Results}

Prognostic significance of MYCN amplification in patients with neuroblastoma

Using the clinical data deposited in TARGET dataset, we analyzed the prognosis of MYCN amplification in patients with neuroblastoma. Consistent with the previous report that MYCN amplification was associated with poor outcome [12], neuroblastoma patients with MYCN amplification had worse prognosis than patients without 
Prognostic significance of MYC target genes: analysis from TARGET dataset
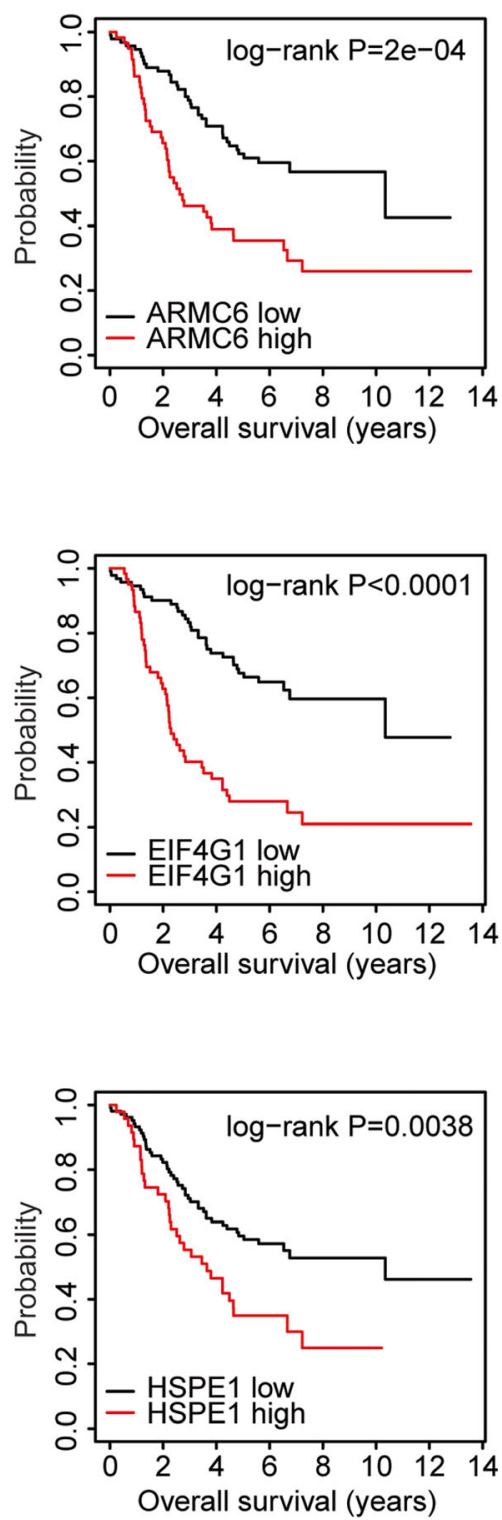
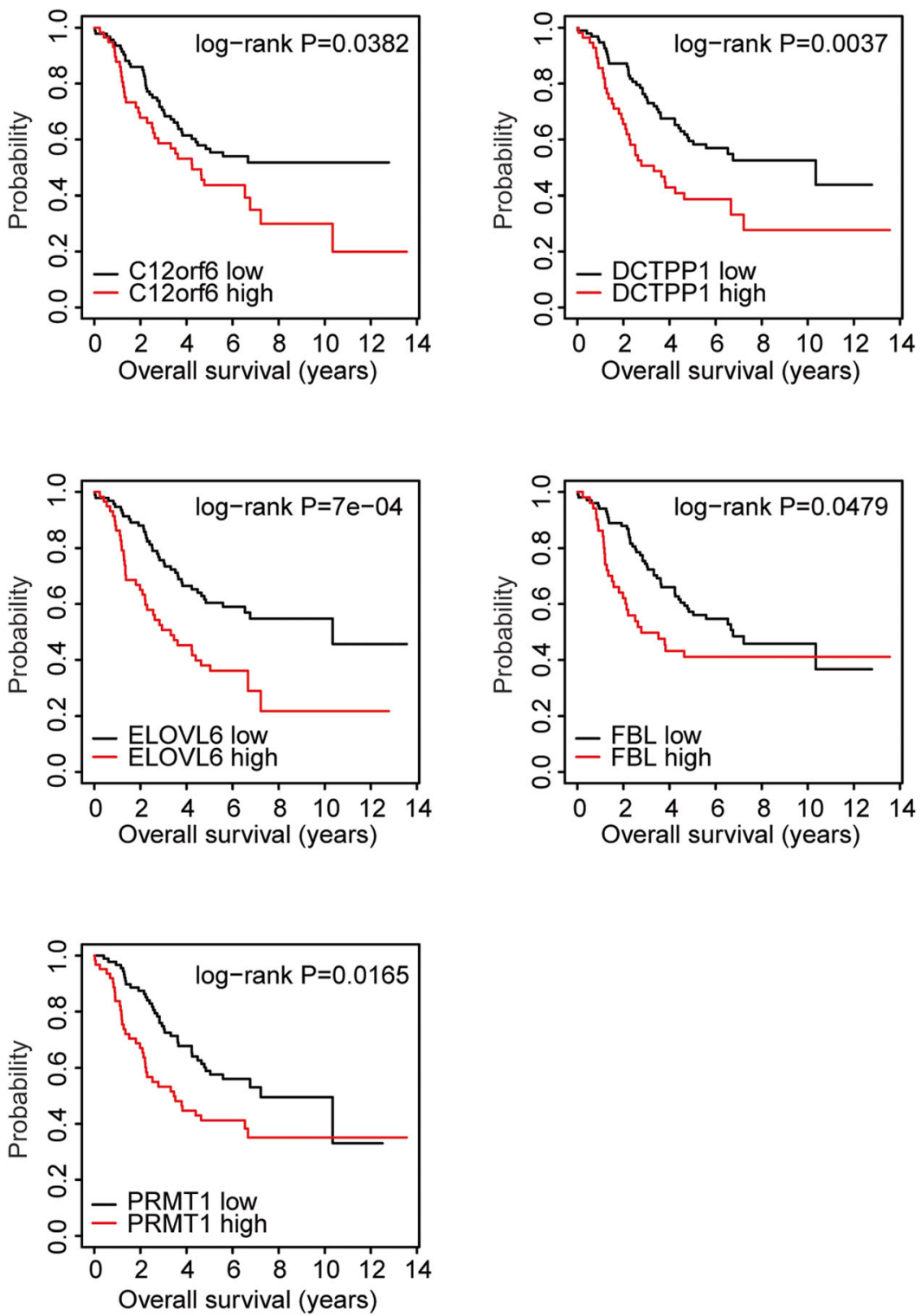

Fig. 3 Prognostic significance of MYCN target genes in patients with neuroblastoma: analysis from TARGET dataset. The Kaplan-Meier plots demonstrated the prognostic effects of MYCN target genes ARMC6, C12orf6, DCTPP1, EIF4G1, ELOVL6, FBL, HSPE1 and PRMT1 in patients with neuroblastoma using TARGET dataset. Patients were divided into two clusters based on the mean expression levels of the MYCN target genes. The log-rank test was used to determine the overall survival $P$-values

MYCN amplification in TARGET dataset (Fig. 1). The prognostic effects of MYCN amplification were further validated in GSE85047 dataset. Neuroblastoma patients with MYCN amplification also demonstrated worse clinical outcomes in GSE85047 dataset (Fig. 1).

\section{Identification of MYCN target genes in patients with neuroblastoma}

To reveal the functional relevance of $\mathrm{MYCN}$ regulated genes in neuroblastoma, we performed transcription factor enrichment analysis through GSEA assay. We found that MYC transcription factor was positively correlated with the MYCN amplification in neuroblastoma in TARGET dataset (Fig. 2a). Transcription factor MYC regulates multiple target genes. In the GSEA assay, we identified 75 MYC target genes which were up-regulated in MYCN amplified neuroblastoma tissues in TARGET dataset (Fig. 2b).

The positive enrichment of MYC transcription factor in MYCN amplified neuroblastoma tissues was also observed in GSE19274 and GSE85047 datasets (Fig. 2a). 
Prognostic significance of MYC target genes: analysis from GSE85047 dataset
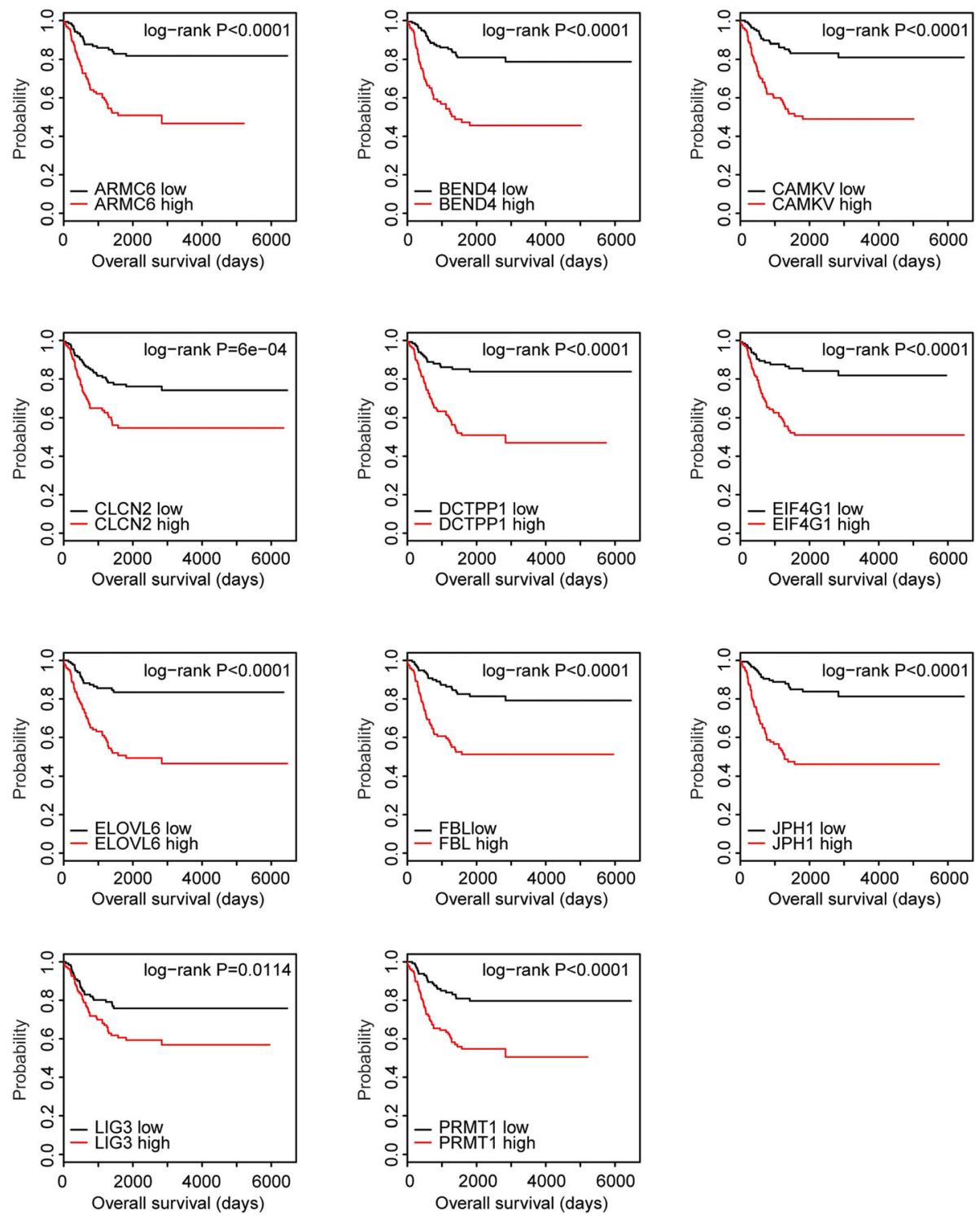

Fig. 4 Prognostic significance of MYCN target genes in patients with neuroblastoma: analysis from GSE85047 dataset. The Kaplan-Meier plots demonstrated the prognostic effects of MYCN target genes ARMC6, BEND4, CAMKV, CLCN2, DCTPP1, EIF4G1, ELOVL6, FBL, JPH1, LIG3 and PRMT1 in patients with neuroblastoma using GSE85047 dataset

We further validated 13 out of 75 MYC target genes ARMC6, BEND4, C12orf66, CAMKV, CLCN2, DCTPP1, EIF4G1, ELOVL6, FBL, HSPE1, JPH1, LIG3 and PRMT1 which were up-regulated in MYCN amplified neuroblastoma tissues in GSE19274 and GSE85047 datasets (Fig. 2b).

Prognostic significance of MYCN target genes in patients with neuroblastoma: analysis from TARGET dataset

Since MYCN amplification was associated with poor outcome in neuroblastoma, we next assessed the prognostic effects of the $13 \mathrm{MYC}$ target genes which were up-regulated in MYCN amplified neuroblastoma. We found that, high expression levels of MYCN target genes ARMC6, C12orf6, DCTPP1, EIF4G1, ELOVL6, FBL, HSPE1 and PRMT1 were associated with worse prognosis in TARGET dataset (Fig. 3). However, other MYCN target genes BEND4, CAMKV, CLCN2, JPH1 and LIG3 demonstrated no prognostic significance.

Prognostic significance of MYCN target genes in patients with neuroblastoma: analysis from GSE85047 dataset The prognostic effects of $13 \mathrm{MYC}$ target genes were further validated in GSE85047 dataset. 11 out of 
the 13 MYC target genes, ARMC6, BEND4, CAMKV, CLCN2, DCTPP1, EIF4G1, ELOVL6, FBL, JPH1, LIG3 and PRMT1 were all correlated with the worse prognosis in patients with neuroblastoma in GSE85047 dataset. Patients with high expression levels of those genes had low overall survival time (Fig. 4). Interestingly, six genes ARMC6, DCTPP1, EIF4G1, ELOVL6, FBL and PRMT1 were associated with the clinical overall survival of neuroblastoma in both TARGET and GSE85047 datasets.
E2F1 is regulated by MYCN amplification and associated with the prognosis of neuroblastoma

Except MYC, transcription factor E2F1 was also positively enriched in MYCN amplified neuroblastoma tissues in GSE19274 and GSE85047 datasets (Fig. 5a). The high expression levels of E2F1 were observed in neuroblastoma patients with MYCN amplification in TARGET, GSE19274, GSE73517, GSE49710 and GSE85047 datasets (Fig. 5b). And high expression of E2F1 was associated with poor prognosis in patients with neuroblastoma in TARGET and GSE85047 datasets (Fig. 5c).

a

Enrichment plots of E2F1 transcription factor
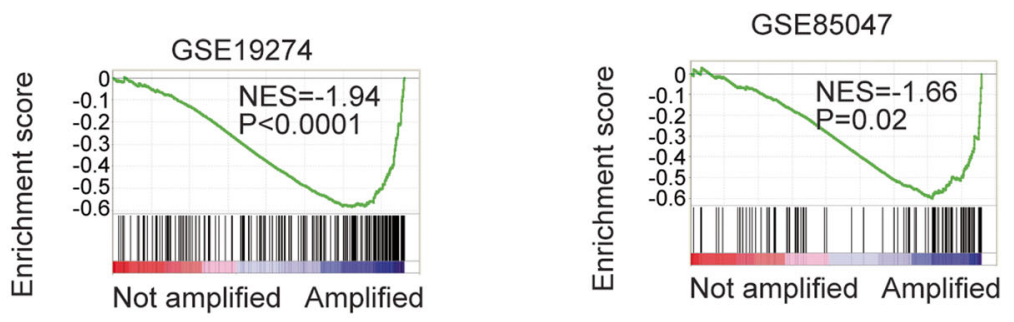

b

TARGET

GSE19274

GSE73517

GSE49710

GSE85047
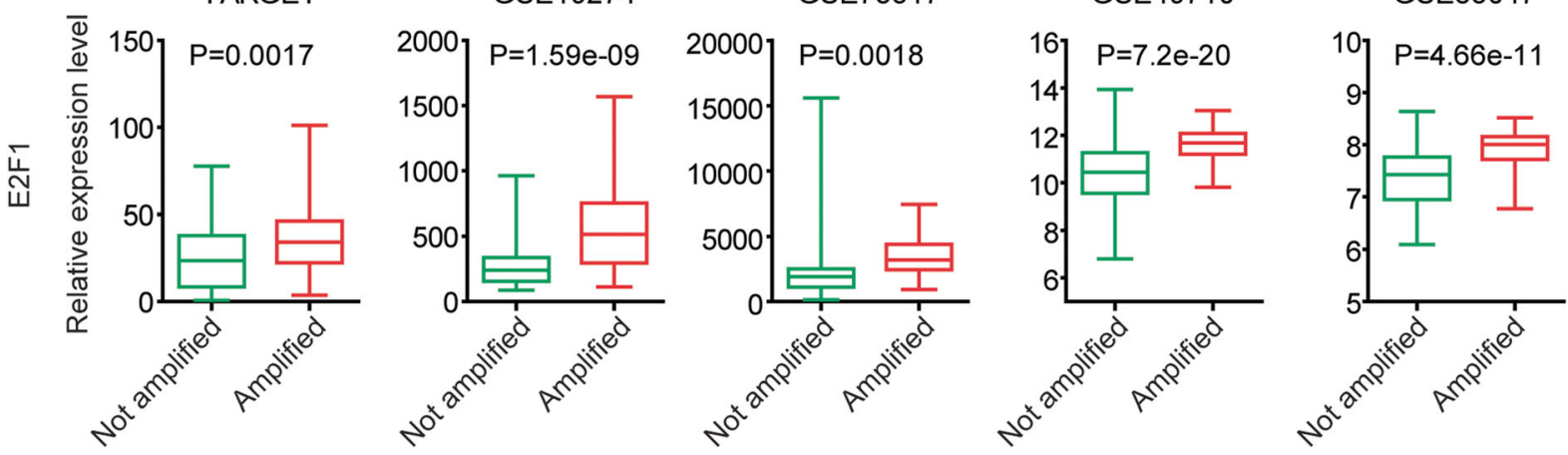

C

TARGET
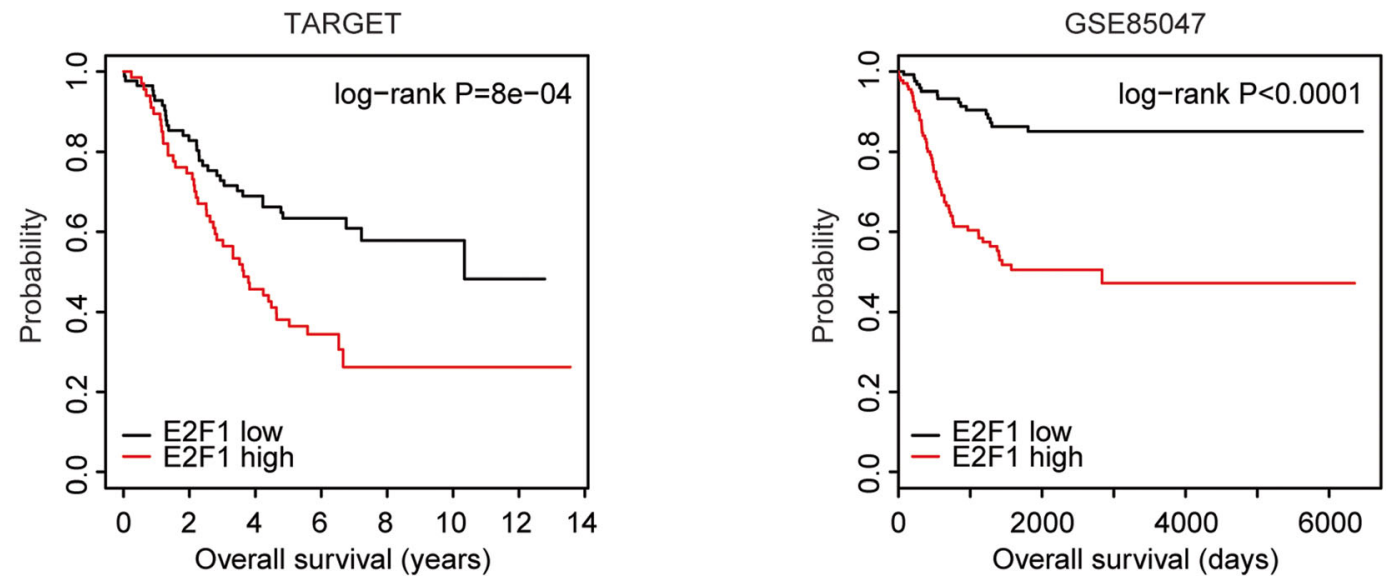

Fig. 5 E2F1 is regulated by MYCN amplification and associated with the prognosis of neuroblastoma. a Enrichment plots of transcription factor E2F1 in GSE19274 and GSE85047 datasets. b Box plots showed the relative E2F1 expression levels in neuroblastoma patients with (red) or without (green) MYCN amplification in TARGET, GSE19274, GSE73517, GSE49710 and GSE85047 datasets. $P$ values were performed using Student's t test. c The Kaplan-Meier plots demonstrated the prognostic effects of E2F1 in patients with neuroblastoma in TARGET and GSE85047 datasets 
RPS19 is regulated by MYCN amplification and associated with the prognosis of neuroblastoma

We also identified the functional signaling pathways which were associated with MYCN amplification in neuroblastoma. We found that ribosome signaling pathway represented the most frequently enriched signaling pathway in TARGET, GSE19274, GSE49710 and GSE85047 datasets (Fig. 6a). Most genes in ribosome signaling pathway were up-regulated in MYCN amplified neuroblastoma patients, as demonstrated the RPS19 expression levels in TARGET, GSE19274, GSE49710,
GSE73517 and GSE85047 datasets (Fig. 6b). However, most genes in ribosome signaling pathway were not associated with the prognosis of neuroblastoma. Only, RPS19 demonstrated poor prognostic effects in patients with neuroblastoma in TARGET and GSE85047 datasets (Fig. 6c).

\section{Correlation of MYCN target genes in patients with neuroblastoma}

So far, we identified eight MYCN target genes ARMC6, DCTPP1, EIF4G1, ELOVL6, FBL, PRMT1, E2F1 and

a

Enrichment plots of Ribosome signaling pathway
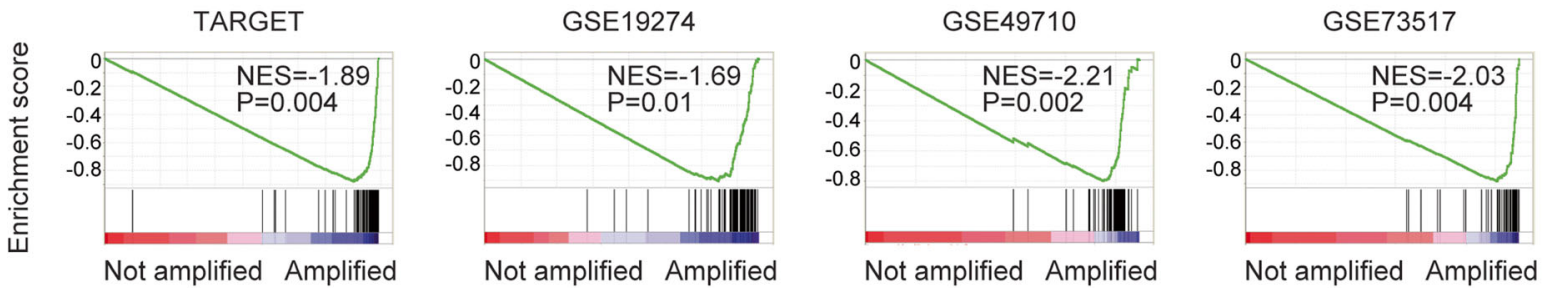

b
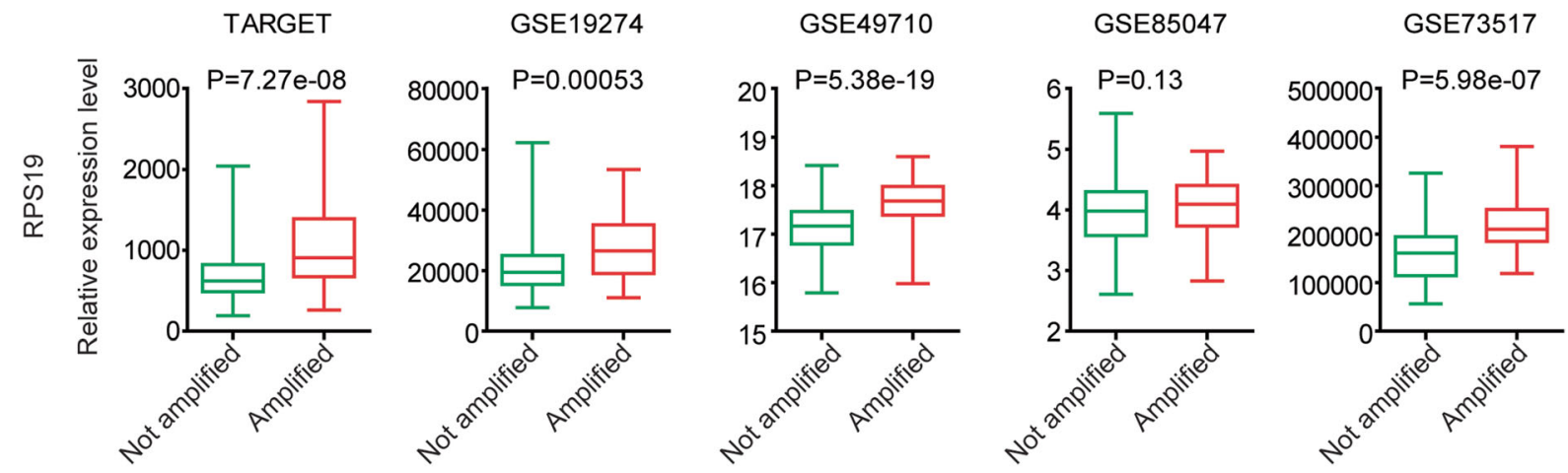

C
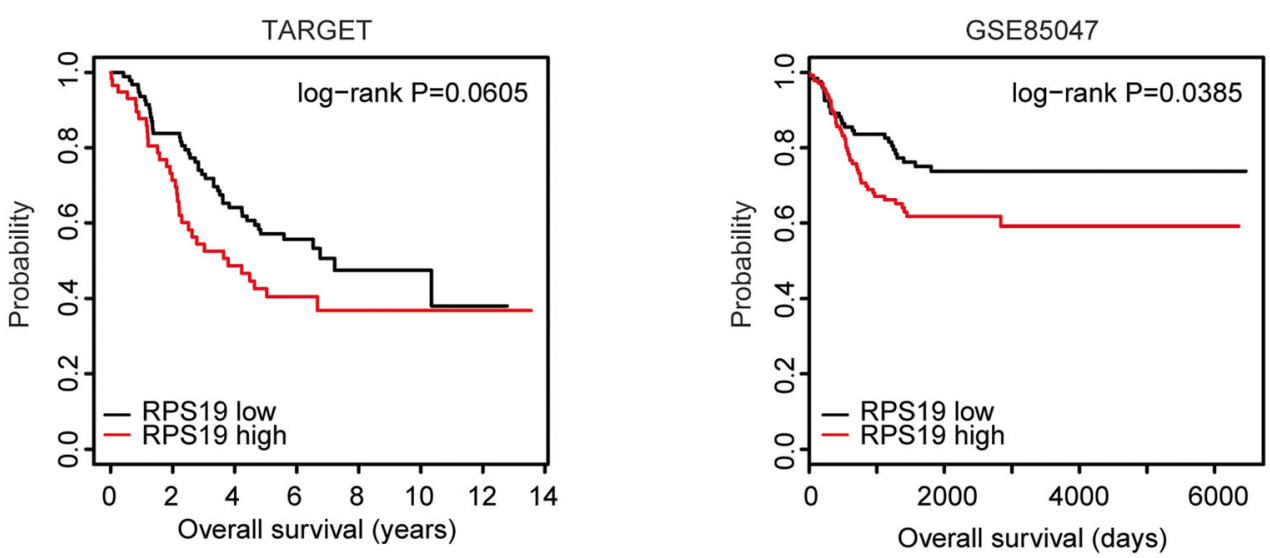

Fig. 6 RPS19 is regulated by MYCN amplification and associated with the prognosis of neuroblastoma. a Enrichment plots of ribosome signaling pathway in TARGET, GSE19274, GSE49710 and GSE85047 datasets. b Box plots showed the relative RPS19 expression levels in neuroblastoma patients with (red) or without (green) MYCN amplification in TARGET, GSE19274, GSE49710, GSE73517 and GSE85047 datasets. c The Kaplan-Meier plots demonstrated the prognostic effects of RPS19 in patients with neuroblastoma in TARGET and GSE85047 datasets 
RPS19 which were up-regulated in MYCN amplified neuroblastoma patients and associated with worse prognosis of neuroblastoma in TARGET and GSE85047 datasets. Next, we determined the correlation of those genes based on their expression levels. ARMC6, DCTPP1, EIF4G1 FBL, PRMT1, E2F1 and RPS19 were highly associated with each other in TARGET dataset (Fig. 7a). However, ELOVL6 was not correlated with other MYCN target genes (Fig. 7a). In GSE85047 dataset, MYCN target genes were correlated with each other except RPS19 (Fig. 7a).

Furthermore, we used multivariate cox regression to determine the association of MYCN target genes in neuroblastoma patients in TARGET and GSE85047 datasets. We found that DCTPP1, EIF4G1 and ELOVL6 were independent prognostic markers in TARGET dataset (Fig. 7b). In
GSE85047 dataset, EIF4G1, ELOVL6 and E2F1 were independent prognostic markers (Fig. 7b). Moreover, MYCN amplification was also an independent prognostic marker in patients with neuroblastoma in GSE85047 dataset (Fig. 7b).

Combined prognostic significance of MYCN amplification and EIF4G1 expression in patients with neuroblastoma In both TARGET and GSE85047 datasets, EIF4G1 was a strong independent prognostic marker. So, we tested the combinational prognostic effects of EIF4G1 expression and MYCN amplification in patients with neuroblastoma. Neuroblastoma patients were divided into four sub-groups based on MYCN status and EIF4G1 mean expression level in TARGET and GSE85047 datasets. Neuroblastoma patients with MYCN amplification and high EIF4G1 expression demonstrated a

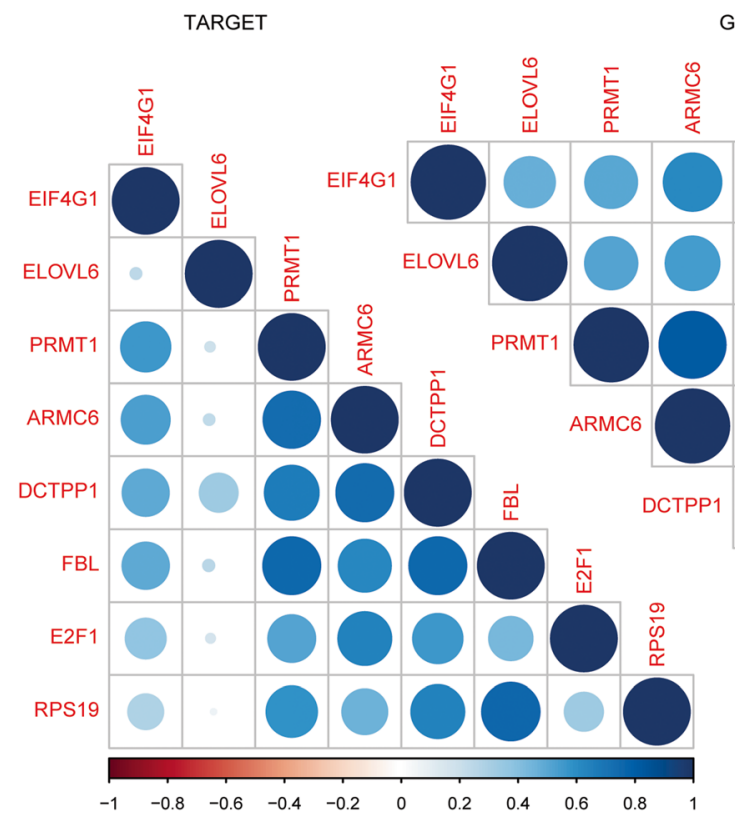

GSE85047

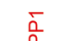


worse clinical outcomes in TARGET dataset (Fig. 8). In GSE85047 dataset, neuroblastoma patients without MYCN amplification were divided into EIF4G1 highly expressed group and EIF4G1 lowly expressed group. We found that neuroblastoma patients without MYCN amplification and with low EIF4G1 expression had best prognosis (Fig. 8).

\section{Discussion}

As the drive alteration in neuroblastoma, prognostic signature associated with MYCN amplification was previously identified. Using MYC target database, results showed that MYC pathway activity was associated with the poor outcome of neuroblastoma [18]. Using neuroblastoma SHEP-21 N cell line with ectopic expression of MYCN [19] or shRNA mediated silencing of MYCN [15], the functional MYCN signatures were revealed. Cohort studies demonstrated the prognostic significance of the MYCN signatures derived from SHEP- $21 \mathrm{~N}$ cell line [20]. However, the overlaps between different prognostic signatures were limited and results from MYCN silencing in neuroblastoma IMR32 cell line demonstrated a completely different prognostic signature [15]. Those observations highlighted the importance of comprehensive and integrated analysis of different cohort of neuroblastoma patients in order to obtain a more precise MYCN related prognostic signature.

So, in the present study, through integrated analysis of TARGET and GEO datasets, we identified eight MYCN amplification associated genes ARMC6, DCTPP1, EIF4G1, ELOVL6, FBL, PRMT1, E2F1 and RPS19 which had significant prognostic effects in pediatric neuroblastoma patients. Previous results showed that PRMT1 regulated MYCN expression in neuroblastoma [21], and down-regulation of PRMT1 induced the senescence of
non-MYCN amplified neuroblastoma cells [22]. E2F1 was a therapeutic target in neuroblastoma. Inhibition of E2F1 suppressed neuroblastoma progression [23]. Inhibition of ribosome singling pathway was also a promising strategy in neuroblastoma treatment [24]. However, the functions and prognosis of ARMC6, DCTPP1, EIF4G1, ELOVL6 and FBL in neuroblastoma were not reported.

The purpose of this study was to determine the transcription factors and signaling pathways associated with MYCN amplification and identify the prognostic relevance of MYCN associated genes in neuroblastoma. The present study suggested new prognostic markers of ARMC6, DCTPP1, EIF4G1, ELOVL6 and FBL in neuroblastoma and highlighted the combinational prognostic significance of MYCN amplification and EIF4G1 expression in patients with neuroblastoma. However, the results were derived from published TARGET and GEO datasets and lack of further validation in neuroblastoma patients. Therefore, functions and prognosis of ARMC6, DCTPP1, EIF4G1, ELOVL6 and FBL in neuroblastoma should be further studied.

\section{Conclusions}

MYC, E2F1 transcription factors and ribosome signaling pathway were significantly enriched in neuroblastoma patients with MYCN amplification. MYCN target genes ARMC6, DCTPP1, EIF4G1, ELOVL6, FBL, PRMT1, E2F1 and RPS19 which had significant prognostic effects in pediatric neuroblastoma patients. Neuroblastoma patients without MYCN amplification and low EIF4G1 expression had best prognosis.
TARGET

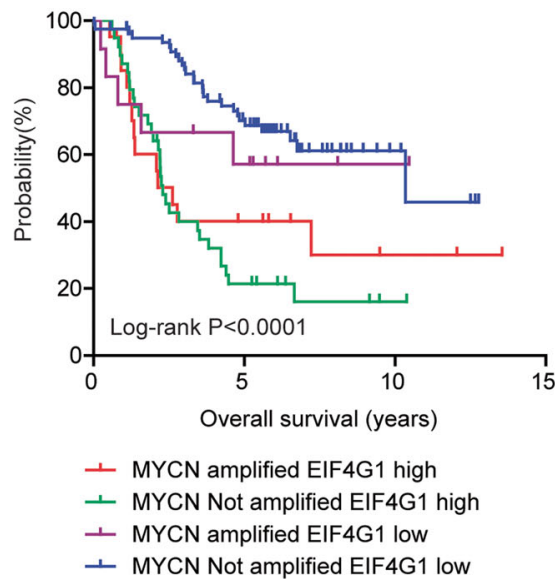

GSE85047

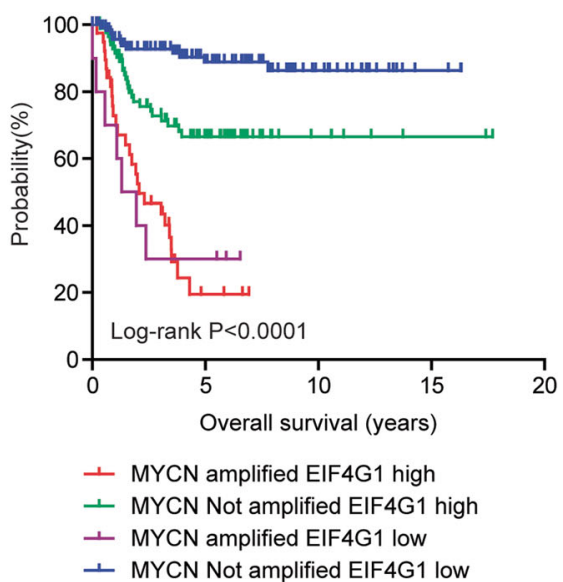

Fig. 8 Combined prognostic significance of MYCN amplification and EIF4G1 expression in patients with neuroblastoma. The Kaplan-Meier plots demonstrated the different overall survival of neuroblastoma patients with different MYCN amplification status and EIF4G1 expression levels in TARGET and GSE85047 datasets. Log-rank test was used to determine the $P$ values 


\section{Abbreviations}

TARGET: Therapeutically Applicable Research to Generate Effective Treatments; GEO: Gene Expression Omnibus;; GSEA: Gene set enrichment analysis; NES: Normalized enrichment score

\section{Acknowledgements}

We appreciate the generosity of the researches from TARGET and GEO groups for sharing the huge amount of data.

\section{Authors' contributions}

HW.W and XR. W designed and performed data analysis. LP. X helped with the data analysis. HW. W wrote the manuscript. JZ and $\mathrm{HC}$ reviewed the manuscript and supervised the work. All authors read and approved the final manuscript.

\section{Funding}

This study was supported by grants from Fujian Maternity and Child Health Hospital (No.YCXB 18-10 and YCXM 19-04). But, the funders had no role in study design, data collection and analysis, decision to publish, or preparation of the manuscript.

\section{Availability of data and materials}

The datasets used and analyzed during the current study are available from the corresponding author on reasonable request.

\section{Ethics approval and consent to participate}

Not applicable.

\section{Consent for publication}

Not applicable.

\section{Competing interests}

The authors declare that they have no competing interests.

Received: 17 April 2020 Accepted: 22 June 2020

Published online: 27 June 2020

\section{References}

1. Maris JM, Hogarty MD, Bagatell R, Cohn SL. Neuroblastoma. Lancet. 2007; 369(9579):2106-20.

2. Maris JM. Recent advances in neuroblastoma. N Engl J Med. 2010;362(23): 2202-11.

3. Brodeur GM. Neuroblastoma: biological insights into a clinical enigma. Nat Rev Cancer. 2003;3(3):203-16

4. Park JR, Eggert A, Caron H. Neuroblastoma: biology, prognosis, and treatment. Hematol Oncol Clin North Am. 2010;24(1):65-86.

5. Look AT, Hayes FA, Shuster JJ, Douglass EC, Castleberry RP, Bowman LC, Smith El, Brodeur GM. Clinical relevance of tumor cell ploidy and N-myc gene amplification in childhood neuroblastoma: a pediatric oncology group study. J Clin Oncol. 1991;9(4):581-91.

6. Ramani P, Nash R, Rogers CA. Aurora kinase a is superior to Ki67 as a prognostic indicator of survival in neuroblastoma. Histopathology. 2015; 66(3):370-9

7. Fardin P, Barla A, Mosci S, Rosasco L, Verri A, Versteeg R, Caron HN, Molenaar JJ, Ora I, Eva A, et al. A biology-driven approach identifies the hypoxia gene signature as a predictor of the outcome of neuroblastoma patients. Mol Cancer. 2010;9:185.

8. Ackermann S, Cartolano M, Hero B, Welte A, Kahlert Y, Roderwieser A Bartenhagen C, Walter E, Gecht J, Kerschke L, et al. A mechanistic classification of clinical phenotypes in neuroblastoma. Science. 2018; 362(6419):1165-70.

9. Campbell K, Gastier-Foster JM, Mann M, Naranjo AH, Van Ryn C, Bagatell R, Matthay KK, London WB, Irwin MS, Shimada H, et al. Association of MYCN copy number with clinical features, tumor biology, and outcomes in neuroblastoma: a report from the Children's oncology group. Cancer. 2017; 123(21):4224-35

10. Irwin MS, Park JR. Neuroblastoma: paradigm for precision medicine. Pediatr Clin N Am. 2015;62(1):225-56

11. Cohn SL, Pearson AD, London WB, Monclair T, Ambros PF, Brodeur GM Faldum A, Hero B, lehara T, Machin D, et al. The international neuroblastoma risk group (INRG) classification system: an INRG task force report. J Clin Oncol. 2009:27(2):289-97.

12. Seeger RC, Brodeur GM, Sather H, Dalton A, Siegel SE, Wong KY, Hammond D. Association of multiple copies of the $\mathrm{N}$-myc oncogene with rapid progression of neuroblastomas. N Engl J Med. 1985;313(18):1111-6.

13. Chan HS, Gallie BL, DeBoer G, Haddad G, Ikegaki N, Dimitroulakos J, Yeger $\mathrm{H}$, Ling V. MYCN protein expression as a predictor of neuroblastoma prognosis. Clin Cancer Res. 1997;3(10):1699-706.

14. Gross N, Balmas Bourloud K, Brognara CB. MYCN-related suppression of functional CD44 expression enhances tumorigenic properties of human neuroblastoma cells. Exp Cell Res. 2000;260(2):396-403.

15. Valentijn $\sqcup$, Koster J, Haneveld F, Aissa RA, van Sluis P, Broekmans ME, Molenaar JJ, van Nes J, Versteeg R. Functional MYCN signature predicts outcome of neuroblastoma irrespective of MYCN amplification. Proc Natl Acad Sci U S A. 2012;109(47):19190-5.

16. Durbin AD, Zimmerman MW, Dharia NV, Abraham BJ, Iniguez AB, WeichertLeahey N, He S, Krill-Burger JM, Root DE, Vazquez F, et al. Selective gene dependencies in MYCN-amplified neuroblastoma include the core transcriptional regulatory circuitry. Nat Genet. 2018;50(9):1240-6.

17. Ma X, Liu Y, Liu Y, Alexandrov LB, Edmonson MN, Gawad C, Zhou X, Li Y, Rusch MC, Easton J, et al. Pan-cancer genome and transcriptome analyses of 1,699 paediatric leukaemias and solid tumours. Nature. 2018:555(7696): 371-6.

18. Fredlund E, Ringner M, Maris JM, Pahlman S. High Myc pathway activity and low stage of neuronal differentiation associate with poor outcome in neuroblastoma. Proc Natl Acad Sci U S A. 2008;105(37):14094-9.

19. Westermann F, Muth D, Benner A, Bauer T, Henrich KO, Oberthuer A, Brors B, Beissbarth T, Vandesompele J, Pattyn F, et al. Distinct transcriptional MYCN/C-MYC activities are associated with spontaneous regression or malignant progression in neuroblastomas. Genome Biol. 2008;9(10):R150.

20. Vermeulen J, De Preter K, Naranjo A, Vercruysse L, Van Roy N, Hellemans J, Swerts K, Bravo S, Scaruffi P, Tonini GP, et al. Predicting outcomes for children with neuroblastoma using a multigene-expression signature: a retrospective SIOPEN/COG/GPOH study. Lancet Oncol. 2009;10(7):663-71.

21. Eberhardt A, Hansen JN, Koster J, Lotta LT Jr, Wang S, Livingstone E, Qian K, Valentijn $\sqcup$, Zheng $Y G$, Schor NF, et al. Protein arginine methyltransferase 1 is a novel regulator of MYCN in neuroblastoma. Oncotarget. 2016:7(39): 63629-39.

22. Lee YJ, Chang WW, Chang CP, Liu TY, Chuang CY, Qian K, Zheng YG, Li C. Downregulation of PRMT1 promotes the senescence and migration of a non-MYCN amplified neuroblastoma SK-N-SH cells. Sci Rep. 2019;9(1):1771.

23. Fang $E$, Wang $X$, Yang F, Hu A, Wang J, Li D, Song H, Hong M, Guo Y, Liu Y, et al. Therapeutic targeting of MZF1-AS1/PARP1/E2F1 Axis inhibits Proline synthesis and neuroblastoma progression. Adv Sci (Weinh). 2019:6(19): 1900581.

24. Hald OH, Olsen L, Gallo-Oller G, Elfman LHM, Lokke C, Kogner P, Sveinbjornsson B, Flaegstad T, Johnsen Jl, Einvik C. Inhibitors of ribosome biogenesis repress the growth of MYCN-amplified neuroblastoma. Oncogene. 2019;38(15):2800-13.

\section{Publisher's Note}

Springer Nature remains neutral with regard to jurisdictional claims in published maps and institutional affiliations.

Ready to submit your research? Choose BMC and benefit from:

- fast, convenient online submission

- thorough peer review by experienced researchers in your field

- rapid publication on acceptance

- support for research data, including large and complex data types

- gold Open Access which fosters wider collaboration and increased citations

- maximum visibility for your research: over $100 \mathrm{M}$ website views per year

At BMC, research is always in progress.

Learn more biomedcentral.com/submissions 